\title{
Erectile Dysfunction Severity Breakpoint
}

National Cancer Institute

\section{Source}

National Cancer Institute. Erectile Dysfunction Severity Breakpoint. NCI Thesaurus. Code C138120.

Scoring groups for the Sexual Health Inventory for Men: 1-7, Severe erectile dysfunction; 8-11, Moderate erectile dysfunction; 12-16, Mild to Moderate erectile dysfunction; 17-21, Mild erectile dysfunction. 